\title{
The patient with head injury and a Glasgow coma score of 15 - is a brain CT examination indicated?
}

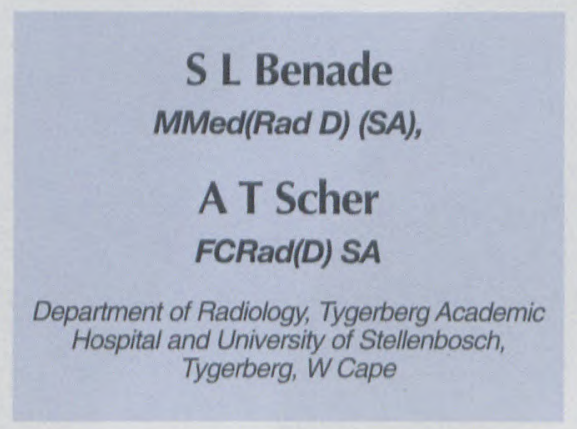

\section{Abstract}

The decision as to whether to perform a CT examination of the brain in patients with a Glasgow coma score of 15 after injury is often difficult, given the limited CT scanning facilities available in state hospitals.

A retrospective evaluation of 100 consecutive head-injury patients presenting with a Glasgow coma score of 15 at Tygerberg Hospital was therefore carried out. In a surprisingly high number of patients $(50 \%)$ abnormal findings due to the injury were detected. Analysis of the clinical history parameters did not demonstrate a significant association with abnormal CT findings. It is therefore concluded that brain CT examination in patients with a Glasgow coma score of 15 is justified, and that the Glasgow coma scale is a poor predictor of intracranial injury.

\section{Introduction}

There is intense debate in the medical literature over whether headinjury patients with a Glasgow coma score of 15 need to be examined by CT. This debate is driven by the need to identify all patients with potentially serious injuries against the limited CT scanning facilities available at many institutions.

The Glasgow coma scale was developed in 1974 as an evaluation instrument to monitor patients with altered consciousness. It is a simple and reproducible examination, utilising three categories of patient response, i.e. the ability to open the eyes, the best verbal response, and the best motor response.

\section{Patients and methodology}

This study is based on the retrospective database evaluation of 100 consecutive head-injury patients (injuries $<36$ hours before admission) referred for a brain CT investi- gation. All patients were 13 years of age or older, and on admission had a Glasgow coma score of 15. The fulltime trauma doctors at the Trauma Unit at Tygerberg Hospital assessed the Glasgow coma score of patients on admission.

\section{Results}

In this study of 100 head-injury patients, the CT examination was abnormal in 56 patients (56\%). In 6 patients the pathology was unrelated to the head injury (Fig 1).

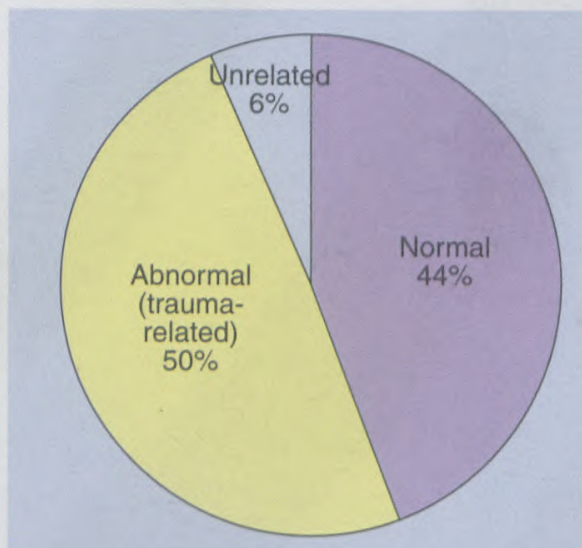

Fig. 1. CT findings for 100 head-injured patients with a Glasgow coma score of 15.

The spectrum of unrelated pathology in the 6 patients was as follows:

- Cysticercosis

- Posterior cerebral artery infarct

- Posterior cerebral artery aneurysm

- Calcified granuloma

- Posterior inferior cerebral artery infarct with subarachnoidal bleeding

- Lacuna infarct of the basal ganglia Mechanisms of injury (Fig. 2):

- 19 motor vehicle accidents (MVAs)

- 6 pedestrians

- 12 passengers

- 1 motorcycle/motorbike cyclist

- 68 assaults

- 7 physical

- 60 object 


\section{ORIGINAL ARTICLE}

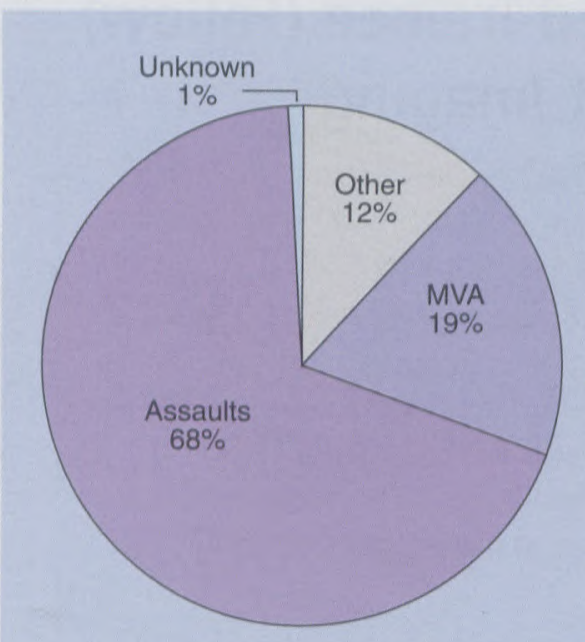

Fig. 2. Mechanism of injury in 100 patients with Glasgow coma score of 15.

- 1 unknown

- 12 Other

- 1 Unknown

\section{Skull X-ray findings}

- $42(84 \%)$ of the 50 patients had single or multiple injuries

- The findings in the 42 patients with fractures were:

- $15(36 \%)$ extra-axial bleeding

- $22(52 \%)$ intra-axial bleeding or contusions

- 22 (52\%) diffuse brain swelling and/or pneumocranium

- $9(16 \%)$ of the 50 patients did not have a fracture, but showed:

- 2 patients had a small subdural bleed

- 1 patient had subarachnoid bleeding

- 5 patients had a contusion

\section{Discussion}

Fifty patients had abnormal CT findings related to their trauma (Figs 3 and 4). This is a very high incidence and there are several reasons for this. One of these is in the design of this study. Most studies in the literature are based on consecutive head-injury patients with a Glasgow coma score of

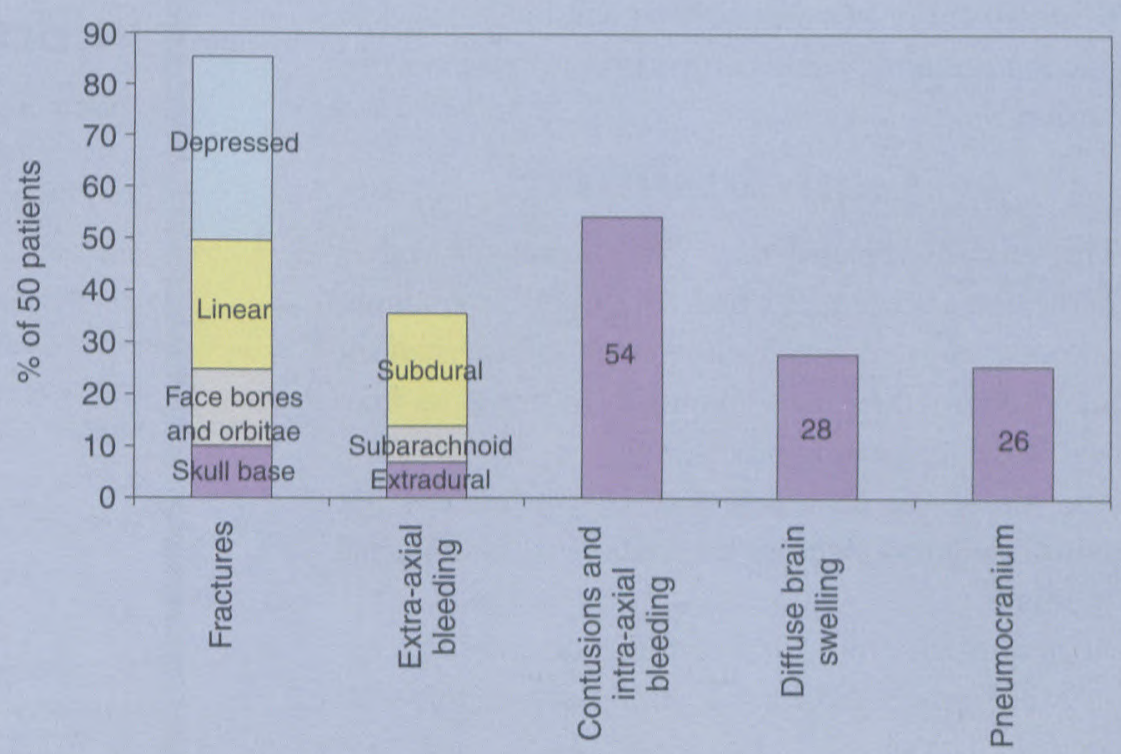

Fig. 3. Findings in 50 patients with an abnormal trauma related CT.

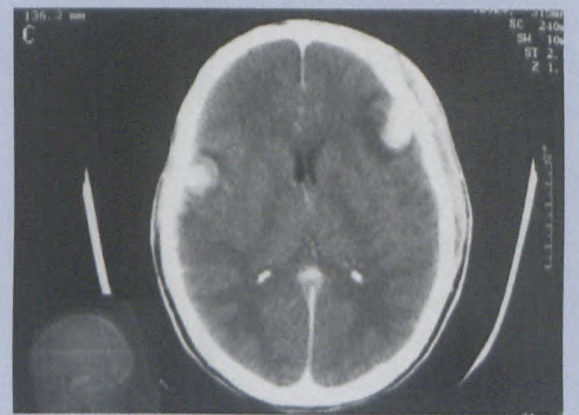

Fig. 4. CT scan of a patient with a Glasgow coma score of 15 who was assaulted. There are right parietal and left frontal cerebral contusions.

15 , all of whom had routine brain CT, while in this study only patients with clinical indications were examined. In comparison, Jeret et al. ${ }^{1}$ found abnormal findings in $9.4 \%$ of 712 consecutive patients with a Glasgow coma score of 15 who were evaluated with brain CT over a 1-year period.

\section{Age}

The average patient age was 31 years (median 28 years). This compared with larger studies in the literature, with a average age of 33 years. ${ }^{2}$

\section{Gender}

Seventy-two percent of patients were male and $28 \%$ were female. This is comparable with other studies which showed that more males than females presented with head injuries in trauma units. ${ }^{3.4}$

\section{Mechanisms of injury}

Sixty-eight patients were assaulted, 19 were MVA patients, 12 had fallen and 1 history was unknown.

In a study of 2766 patients, Shackford et al..$^{5}$ identified assault as the mechanism of injury in only $8 \%$ of patients. Livingston et al. ${ }^{6}$ showed that as a mechanism of injury assault was associated with a higher incidence of intracranial pathology. The very high percentage of assaults in this study in comparison with the literature is probably the reason for the high number of abnormal CTs.

\section{Clinical history}

In the patient group with abnormal CT findings, $96 \%$ had headache, 
$34 \%$ nausea and vomiting, $22 \%$ loss of consciousness, $10 \%$ had memory loss and $10 \%$ convulsions. No clear distinction was observed in comparison with the normal CT group.

\section{Conclusion}

This analysis revealed that $56 \%$ of patients with a Glasgow coma score of 15 had abnormal CT findings, many of which were significant. Assault as a mechanism of injury occurred far more frequently in this series than in large series analysed in other countries.

Analysis of the clinical history parameters did not demonstrate any significant association with abnormal CT findings.

An abnormal neurological examination and suspicion of a skull fracture did have a strong association with abnormal CT findings.

In $84 \%$ of patients with one or more fractures, the CT examination was abnormal.

In light of the above findings it can be concluded that brain CT examination in patients with a Glasgow coma score of 15 is justified, and that the Glasgow coma scale is a poor predictor for normal brain CT examination.

\section{References}

1. Jeret JS, Mandell M, Anziska B, et al. Clinical predictors of abnormality disclosed by computed tomography after mild head trauma. Neurosurgery 1993; 32(1): 9-16.

2. Nagy KK, Joseph KT, Krosner SM, et al. The utility of head computed tomography after minimal head injury. J Trauma 1999; 46: 268 -270.

3. Miller EC, Holmes JF, Derlet RW. Utilizing clinical factors to reduce head CT scan ordering for minor head trauma patients. J Emerg Med 1997; 15: 453-457

4. Stiell IG, Wells GA, Vandemheen K, et al. Variation in ED use of computed tomography for patients with minor head injury. Ann Emerg Med 1997; 30(1): 14-22.

5. Shackford SR, Wald SL, Ross SE, et al. The clinical utility of computed tomography scanning and neurologic examination in the management of patients with minor head injuries. J Trauma 1992; 33: 385-393.

6. Livingston DH, Lader PA, Koziol J, Hunt CD. The use of CT scanning to triage patients requiring admission following minimal head injury. $J$ Trauma 1991; 31: 483-489.
Royal Perth Hospital

\section{Advanced Trainee (Fellow) in Breast Imaging}

Applications are invited from medical practitioners who are fully registrable in Western Australia to fill the above position from 19 January 2004 to 16 January 2005.

Applicants should possess the Part II DRACR or equivalent and wish to gain further experience in breast imaging. Five (5) sessions will be spent in Breast Imaging and the remaining 5 sessions in general cross sectional imaging. Royal Perth Multi-disciplinary Breast Service provides screening and diagnostic clinics, as well as a pre-operative hookwire localisation service.

Royal Perth Hospital is a 955 bed major teaching hospital. The Radiology Department provides a full range of diagnostic and interventional radiological services to the Hospital and to Royal Perth Rehabilitation Hospital and the Spinal Unit.

The Department has two DSA units, a superconductive 1.0 Tesla magnetic resonance scanner, two Siemens CT scanners, and modern ultrasound equipment (including colour Doppler).

The appointee will be expected to assist with undergraduate and postgraduate teaching programs, applicants are encouraged to participate in research projects and also participate in the Departmental on-call roster.

Royal Perth Multi-disciplinary Breast Service runs a busy screening and diagnostic breast imaging service. Extensive experience in image-guided biopsy including Mammotome vacuum assisted biopsy will be available as well as experience gained in pre-operative hookwire localisation.

Terms and Conditions of Employment are in accordance with the Medical Practitioners (Metropolitan Health Services) AMA Industrial Agreement 2002. The salary range currently for this position is $A \$ 64,975$ - $A \$ 98,940$ per annum together with a Professional Expenses Allowance of A\$1,949 per annum. Point of commencement in the salary range is dependent. upon qualifications and experience.

Further details regarding this position may be obtained from Professor Mark Khangure, Head of Department, Diagnostic and Interventional Radiology, Royal Perth Hospital on (+61 8) 9224 2126, facsimile (+61 8) 9224 2912, e-mail: mark.khangure@health.wa.gov.au or Dr Elizabeth Wylie, Department of Diagnostic and Interventional Radiology, Royal Perth Hospital on (+61 8) 92242125 , facsimile (+61 8) 92242912 or e-mail: liz.wylie@health.wa.gov.au Applications stating qualifications, previous experience and the names and addresses of two professional referees should be forwarded to Joyce O'Hara, Senior Administrative Assistant, Clinical Services, Royal Perth Hospital, GPO Box X 2213, Western Australia 6847.

Closing Date: Friday 29 August 2003. 